\title{
MicroRNA-1915-3p inhibits cell migration and invasion by targeting SET in non-small- cell lung cancer
}

Hongli Pan ${ }^{1}$, Zhenhua Pan ${ }^{1}$, Fengjie Guo ${ }^{1}$, Fanrong Meng ${ }^{2}$, Lingling $\mathrm{Zu}^{1}$, Yaguang Fan', Yang Li ${ }^{1}$, Mengjie Li ${ }^{3}$, Xinxin Du', Xiuwen Zhang ${ }^{4,5}$, Yi Shao ${ }^{6}$, Mingming Wei ${ }^{7^{*}}$, Xuebing $\mathrm{Li}^{1^{*}}$ and Qinghua Zhou ${ }^{1,3^{*}}$

\begin{abstract}
Background: MicroRNAs (miRNAs) have been reported to play significant roles in non-small-cell lung cancer (NSCL C). However, the roles of microRNA (miR)-1915-3p in NSCLC remain unclear. In this study, we aimed to explore the biological functions of miR-1915-3p in NSCLC.

Methods: The expression of miR-1915-3p and SET nuclear proto-oncogene (SET) in NSCLC tissues were examined by quantitative real-time PCR (qRT-PCR). Migratory and invasive abilities of lung cancer were tested by wound healing and transwell invasion assay. The direct target genes of miR-1915-3p were measured by dual-luciferase reporter assay and western blot. Finally, the regulation between METTL3/YTHDF2/KLF4 axis and miR-1915-3p were evaluated by qRT-PCR, promoter reporter assay and chromatin immunoprecipitation (CHIP).

Results: miR-1915-3p was downregulated in NSCLC tissues and cell lines, and inversely associated with clinical TNM stage and overall survival. Functional assays showed that miR-1915-3p significantly suppressed migration, invasion and epithelial-mesenchymal transition (EMT) in NSCLC cells. Furthermore, miR-1915-3p directly bound to the 3' untranslated region (3'UTR) of SET and modulated the expression of SET. SET inhibition could recapitulate the inhibitory effects on cell migration, invasion and EMT of miR-1915-3p, and restoration of SET expression could abrogate these effects induced by miR-1915-3p through JNK/Jun and NF-kB signaling pathways. What's more, miR1915-3p expression was regulated by METTL3/YTHDF2 m6A axis through transcription factor KLF4.
\end{abstract}

Conclusions: These findings demonstrate that miR-1915-3p function as a tumor suppressor by targeting SET and may have an anti-metastatic therapeutic potential for lung cancer treatment.

Keywords: NSCLC, miR-1915-3p, Invasion, Migration, SET

\footnotetext{
* Correspondence: mingmingshengwu@163.com; xbli@tmu.edu.cn;

zhouqh1350@126.com

${ }^{7}$ The State Key Laboratory of Medicinal Chemical Biology, College of

Pharmacy, Nankai University, Tianjin, China

'Tianjin Key Laboratory of Lung Cancer Metastasis and Tumor

Microenvironment, Tianjin Lung Cancer Institute, Tianjin Medical University

General Hospital, Tianjin, China

Full list of author information is available at the end of the article
}

(C) The Author(s). 2021 Open Access This article is licensed under a Creative Commons Attribution 4.0 International License, which permits use, sharing, adaptation, distribution and reproduction in any medium or format, as long as you give appropriate credit to the original author(s) and the source, provide a link to the Creative Commons licence, and indicate if changes were made. The images or other third party material in this article are included in the article's Creative Commons licence, unless indicated otherwise in a credit line to the material. If material is not included in the article's Creative Commons licence and your intended use is not permitted by statutory regulation or exceeds the permitted use, you will need to obtain permission directly from the copyright holder. To view a copy of this licence, visit http://creativecommons.org/licenses/by/4.0/ The Creative Commons Public Domain Dedication waiver (http://creativecommons.org/publicdomain/zero/1.0/) applies to the data made available in this article, unless otherwise stated in a credit line to the data. 


\section{Background}

Lung cancer is the second most frequent cancer and the leading cause of cancer-related fatality worldwide, with 2.21 million new cases (approximately $11.4 \%$ of total) and 1.80 million deaths (almost $18.0 \%$ of total) estimated in 2020 [1]. Approximately 85\% of lung cancers is nonsmall-cell lung cancer (NSCLC), associated with a poor prognosis [2]. Although extensive research and progress in the early diagnosis and targeted therapies of lung cancer have a rapid development in recent decades, metastasis is still the main challenge posed by advanced lung cancer resulting in a high mortality [3]. Previous studies have verified that more than $50 \%$ of NSCLC patients present with metastasis [4]. Therefore, further investigations should focus on the molecular mechanism of NSCLC metastasis.

MicroRNAs (miRNAs) are a kind of small endogenous non-coding RNAs with 18-25 nucleotides in length, which can alter mRNA stability and/or translation efficiency of target genes by perfect or imperfect basepairing with the 3'untranslated regions (UTRs) [5], 5' UTRs [6] or open reading frames (ORFs) region [7] of target mRNAs. Accumulating evidence have shown that miRNAs participate in a wide range of biological processes in several types of cancers [8-10]. In NSCLC, many miRNAs have been proved to function as oncogenes or tumor suppressors. For instance, miR-21, acting as an oncogene, suppressed cell apoptosis and promoted NSCLC tumorigenesis through inhibition of negative regulators of the Ras/MEK/ERK pathway in vitro and in vivo [11]. In contrast, miR-34 was downregulated in NSCLC and modulated the expression of proteins involved in cell cycle, anti-apoptosis, tumor metastasis and immune invasion [12,13], served as a tumor suppressor. Moreover, several studies revealed that miRNA mimics or inhibitors have potential as anticancer therapeutics $[14,15]$. However, only few miRNA therapeutics have so far moved into clinical development. The absence of tumor-specific miRNA therapeutic options for NSCLC underscores the critical need to achieve a better understanding of the biological roles of miRNAs in lung cancer, thus developing better therapeutic strategies for NSCLC patients.

In our previous research, in order to achieve a better understanding of the role of miRNAs in lung cancer, we conducted miRNA microarray in 10 paired NSCLC tissues and found that miR-1915-3p was one of the most significantly downregulated miRNAs in NSCLC tissue samples [16]. Several studies have shown that miR-1915$3 \mathrm{p}$ modulates multidrug resistance, anti-apoptosis and stem cell differentiation by targeting BCL2 apoptosis regulator (BCL2) [17-19], prominin 1 (PROM1) and paired box 2 (PAX2) [20] in multiple types of cancers. Although several miR-1915-3p's target genes have been confirmed, the underlying mechanisms of the involvement of miR-1915-3p and its targets in NSCLC metastasis are still not fully understood.

In this study, we investigated whether miR-1915-3p expression was associated with clinicopathological parameters, whether miR-1915-3p modulated the metastatic abilities of NSCLC cells by altering its targets expression, and whether miR-1915-3p expression was regulated by $\mathrm{N}^{6}$-methyladenosine (m6A) modification.

\section{Methods}

\section{Patients and tissue specimens}

This study was approved by the ethics committee of Tianjin Medical University General Hospital (Tianjin, China). In our study, we used 73 paired NSCLC tissues. We also obtained written informed consent from the 73 NSCLC patients. All of these patients were not received any chemotherapy or radiotherapy before surgery. Tumor and corresponding normal lung tissue samples were taken at the time of surgery and rapidly frozen in liquid nitrogen. The tumor tissues contained a tumor cellularity of greater than $60 \%$ and the matched control tissues had no tumor content. The clinicopathological characteristics of all patients were collected and analyzed.

\section{Cell culture and transfection}

The NSCLC cell lines (A549 and H1975) were cultured in F-12 K or RPMI-1640 medium supplemented with $10 \%$ FBS (Hyclone, USA) at $37{ }^{\circ} \mathrm{C}$ and $5 \% \mathrm{CO}_{2}$.

Negative control, miR-1915-3p mimic, anti-miR-1915-3p were synthesized by GenePharma (Shanghai, China). SiNC (cat. no. sc-37,007), siSET (cat. no. sc-43,856), siMETTL3 (cat. no. sc-92,172), siYTHDF2 (cat. no. sc-78,661) and siKLF4 (cat. no. sc-35,480) were purchased from Santa Cruz biotechnology (Dallas, Texas, USA). The sequences of these oligonucleotides were as follows: miR-NC: $5^{\prime}$ UUCUCCGAACGUGUCACGUTT-3'; miR-1915-3p mimic: 5 '-CCCCAGGGCGACGCGGCGGG-3'; anti-miR1915-3p: 5'-CCCGCCGCGUCGCCCUGGGG-3'; antimiR-NC: 5' -CAGUACUUUUGUGUAGUACAA-3'.

For SET overexpression, SET were amplified from H1975 cDNA, and subcloned downstream of FLAG tag in the pcDNA3.1-flag vector. The primers used for the coding sequences of SET amplification were as follows: 5'-GCGCGAATTC (EcoR I) ATGTCGGCGCCGGCGG CCAA-3' (forward primer) and 5' -GCGCCTCGAG (Xho I) 'TTAGTCATCTTCTCCTTCATCCTC-3' (reverse primer). For human KLF4 overexpression, the plasmid FLAG-KLF4 was generated, and the primers were as follows: 5'-GCGCGAATTC (EcoR I) ACATTAATGAGG CAGCCACCTG-3' (forward primer) and 5'-GCGCGG ATCC (BamH I) CGGGGGATTTAAAAATGCCTC-3' (reverse primer). 
Transient transfection of A549 or H1975 cells were performed using Lipofectamine 2000 (Invitrogen, USA).

\section{Quantitative RT-PCR}

The expression level of related genes was measured by quantitative real-time PCR (qRT-PCR). We used the $2^{-\Delta \Delta C T}$ method to calculate the fold changes of mRNA or miRNA expression. The primers used for qRT-PCR were presented in Table 1. The primers of miR-1915-3p and RNU6B were purchased from Qiagen. The primers used for qRT-PCR were synthesized by BGI (BGI, China).

\section{Wound healing assay}

For the wound healing assay, A549 and H1975 cells were seeded into six-well plates and grown to $100 \%$ confluence. Wounds were created in the middle of the cell monolayer and assessed by phase-contrast microscope at indicated time points. Wound width was measured from four different positions. Relative wound width was

Table 1 The primers used for qRT-PCR

\begin{tabular}{|c|c|}
\hline Gene & Primer $\left(5^{\prime}-3^{\prime}\right)$ \\
\hline \multirow[t]{2}{*}{ C1RL } & F: ATCTCATTCGTCGGTTCG \\
\hline & R: TGGGCAGTCTTGTTCTCC \\
\hline \multirow[t]{2}{*}{ CHTF8 } & F: TCGCTACAGCACTGGATTA \\
\hline & R: GGACGCTGGTGATAATGG \\
\hline \multirow[t]{2}{*}{ MMP24 } & F: GGCAAAAACACATCACCTAC \\
\hline & R: GGTCACTITTGATCTCATGG \\
\hline \multirow[t]{2}{*}{ PPP2R5D } & F: САTCTCTTCCСTTTCCTTCATTCG \\
\hline & R: AGCCACTGGAACAAGACAATCC \\
\hline \multirow[t]{2}{*}{ SET } & F: CGAAGTCCACCGAAATCAAATGG \\
\hline & R: TCAGAATGGTCAGTAAACCAGGT \\
\hline \multirow[t]{2}{*}{ TFE3 } & F: TGCTCCATCCTITGTCTTG \\
\hline & R: GCTGGGTCTCATCCTCACT \\
\hline \multirow[t]{2}{*}{ TSKU } & F: CCCGAGTAACTTATGTTCAATG \\
\hline & R: GACCCGAGTCTGGTTTGG \\
\hline \multirow[t]{2}{*}{ KLF4 } & F: TCGGGCGGCTTCGTGGCCGA \\
\hline & R: CGTACTCGCTGCCAGGGGCG \\
\hline \multirow[t]{2}{*}{ METTL3 } & F: CAAGCTGCACTTCAGACGAA \\
\hline & R: GCTTGGCGTGTGGTCTTT \\
\hline \multirow[t]{2}{*}{ YTHDF2 } & F: TAGCCAACTGCGACACATTC \\
\hline & R: CACGACCTTGACGTTCCTTT \\
\hline \multirow[t]{2}{*}{ GAPDH } & F: GAGTCAACGGATTTGGTCGT \\
\hline & R: GACAAGCTTCCCGTTCTCAG \\
\hline \multirow[t]{2}{*}{ Primary (pri) miR-1915 } & F: TGTCCCCTTCTCTCCAGCT \\
\hline & R: GCTGCAGAGGCAGGGCTTTC \\
\hline \multirow[t]{2}{*}{ Precursor (pre) miR-1915 } & F: AGGCCGCACCTTGCCTT \\
\hline & R: TGGGGCCCACGGGTGCA \\
\hline
\end{tabular}

$F$ Forward, $R$ Reverse calculated as the ratio between the final and the initial actual wound width.

\section{Cell invasion assay}

Cell invasion assay was conducted by using Matrigel Invasion Kits (Corning, USA). Transwell inserts were rehydrated for at least $2 \mathrm{~h}$. Transfected cells $\left(2 \times 10^{4}\right.$ cells $)$ were resuspended in serum-free medium and then placed into the upper chamber, while complete medium containing 10\% FBS was added in the bottom chamber as a chemoattractant. $24 \mathrm{~h}$ later, cells were fixed by methanol and stained with crystal violet. The noninvading cells on the upper surface were removed by wiping with a cotton swab. The invading cells were counted through microscope. At least five fields were counted from each chamber to assess the invasive property of cells. All assays were repeated independently in triplicates.

\section{3'UTR dual-luciferase reporter assay}

The 3'UTR sequences of SET including miR-1915-3p binding sites were obtained from A549 cDNA, termed SET-3UTR. SET-3UTR-Del excluding miR-1915-3p binding sites were synthetized from BGI (BGI, Shenzhen, China). SET-3UTR and SET-3UTR-Del sequences were then subcloned downstream of the firefly luciferase gene in the modified pGL3-control vector, respectively.

For dual-luciferase reporter assay, cells were cotransfected with $0.2 \mu \mathrm{g}$ of the constructed pGL3-control reporter, $20 \mathrm{nM}$ of the miR-1915-3p mimic or miR-NC, and $0.02 \mu \mathrm{g}$ of Renilla expressing plasmid as an internal control by using Lipofectamine 3000 (Invitrogen). After transfection for $48 \mathrm{~h}$, luciferase activities were measured using the dual-luciferase reporter assay system (Promega, USA). The ratio between firefly and Renilla luciferase activity represented relative firefly luciferase activity.

\section{Promoter reporter and dual luciferase assay}

A 1228 bp fragment DNA containing two KLF4 binding sites from -1005 to +223 relative to the transcription start sites of miR-1915 was amplified from A549 genome and subcloned into pGL3-Basic vector. Deletion reporters (Del-1, Del-2, Del-1/2) were then generated.

For promoter reporter assay, cells were transfected with miR-1915 promoter reporter or KLF4 overexpression plasmid and Renilla as internal control. After transfection for $48 \mathrm{~h}$, dual luciferase assay was performed according to the manufacturer's protocol.

\section{Chromatin immunoprecipitation assay}

Cells $\left(1 \times 10^{7}\right)$ were fixed with paraformaldehyde for 10 $\mathrm{min}$, and then neutralized with Glycine for $5 \mathrm{~min}$. Cells were washed and harvested with PBS, and prepared for a chromatin immunoprecipitation (ChIP) assay using 
Magna CHIP kit (Millipore, USA). For KLF4 CHIP, IgG CHIP were performed as control. Purified DNA was then analyzed by PCR to amplify a $215 \mathrm{bp}$ fragment with the primers as follows: forward, 5'-CCTGGTCACAAA GTACGTGC-3' and reverse, 5'-CTGAGCTGGCCG CCAGCTC-3'. The PCR products were electrophoresed on a $2.5 \%$ agarose gel and stained with ethidium bromide.

\section{Protein extraction and western blotting}

Cells were lysed on ice in RIPA buffer (Beyotime Biotechnology, China). Approximately $20 \mu \mathrm{g}$ protein samples was separated in $12 \%$ SDS-PAGE and electroblotted onto a nitrocellulose membrane (Pall, USA). After blocking by $5 \%$ BSA in Tris-buffered saline with $1 \mathrm{~h}$, the nitrocellulose membrane was hybridized with primary antibodies against anti-SET (cat. no. 55201-1-AP) from Proteintech (Rosemont, USA); anti-E-cadherin (cat. no. 14472), Vimentin (cat. no. 5741), c-Jun (cat. no. 9165), phospho-c-Jun (Ser63) (cat. no. 91952), JNK (cat. no. 9252), phosphorJNK (Thr183/Tyr185), NF-kB pathway antibody sampler kit (cat. no. 4888), METTL3 (cat. no. 86132) from CST (Danvers, USA); N-cadherin antibody (cat. no. A0433) from ABclonal (Wuhan, China); KLF4 antibody (cat. No. sc-393,462) from Santa Cruz biotechnology (Dallas, Texas, USA); FLAG antbody (cat. no. F1804), anti- $\beta$-actin (cat. no. A3853) from Sigma (St. Louis, USA). Blots were also incubated with HRP-linked secondary antibodies that were either anti-mouse IgG (cat. no. 7076) or anti-rabbit IgG (cat. no. 7074), and detected using GeneGnome XRQ Chemiluminescence Image System (Gene Company Limited, China). Densitometric quantification of protein bands was conducted using Image software and then normalized to beta-actin.

\section{Statistical analysis}

Experimental data were presented as mean \pm standard error of mean (SEM). The Chi-square test was used to determine the correlation between miR-1915-3p expression and clinicopathological parameters. Differences between groups were estimated using Student's t test (normal distribution data) or Wilcoxon rank sum test (non-normal distribution data). The association between miR-1915-3p and SET expression was analyzed by spearman's rank correlation. All statistical analyses were carried out using SPSS version 22.0 software (IBM, USA).

\section{Results}

MiR-1915-3p is downregulated in NSCLC tissues and inversely associates with prognosis

In our previous research, in order to identify differentially expressed miRNAs between NSCLC tissues and paired adjacent lung tissues, we performed miRNA microarray [16]. We found that miR-1915-3p was one of the most significantly downregulated miRNAs in NSCL
C tissue samples. MiR-1915-3p expression in 8/10(80\%) NSCLC patients was lower than in the paired adjacent lung tissues (Fig. 1A). Next, our results showed that miR-1915-3p expression was decreased by at least 2-fold in $32 / 73(43.84 \%)$ tumor tissues compared to matching normal lung samples (Fig. 1B). These patients were divided into two subgroups according to the relative expression of miR-1915-3p ( $\log _{2}$ fold change of tumor/ normal $<0$ or $>0$ ). The correlation between miR-1915-3p expression and age, gender, smoking history, histology, TNM classification, EGFR status were then analyzed. The expression of miR-1915-3p was significantly inversely correlated with clinical TNM stage (Table 2). Patients with lower miR-1915-3p expression had a more advanced TNM classification (Fig. 1C). Moreover, we further evaluated the relationship between miR-1915-3p expression and overall survival (OS) in NSCLC. Using the online bioinformatics tool Kaplan-Meier plotter [21], we found that patients with a decreased miR-1915-3p expression had much shorter overall survival compared with patients expressing increased miR-1915-3p levels (Fig. 1D). Taken together, these results revealed that miR-1915-3p was downregulated in NSCLC tissues and inversely associated with clinical TNM stage and OS, suggesting that miR-1915-3p may function as a tumor suppressor in NSCLC development.

\section{MiR-1915-3p inhibits migration, invasion and EMT of NSCLC cells}

Considering that miR-1915-3p expression was inversely correlated with TNM stage, we speculated that miR1915-3p might play a key role in cell migration and invasion. Initially, we examined miR-1915-3p expression levels in eleven lung cancer cell lines (H1975, H23, H1299, PC9, 95D, L78, HCC827, H460, SK-MES-1, H446 and A549) and a human normal bronchial epithelial cell line (BEAS-2B). Our data showed that the expression of miR-1915-3p was lower in lung cancer cell lines compared with normal bronchial epithelial cell (Fig. 2A). Among these lung cancer cell lines, we selected A549 (with the lowest expression of miR-19153p) and H1975 (with the highest expression of miR1915-3p) for further research.

To explore the important role of miR-1915-3p in migration and invasion, a miR-1915-3p mimic or inhibitor were transfected into A549 and H1975 cells using Lipofectamine2000, respectively (Fig. 2B, C). Migration and invasion assays were then performed. These results showed that miR-1915-3p overexpression obviously suppressed migratory and invasive abilities of A549 and H1975 cells (Fig. 2D, F), whereas miR-1915-3p knockdown drastically elevated NSCLC cell migration and invasion (Fig. 2E, G).

Moreover, we tested the expression of EMT-specific markers in transfected NSCLC cells. Notably, the protein 


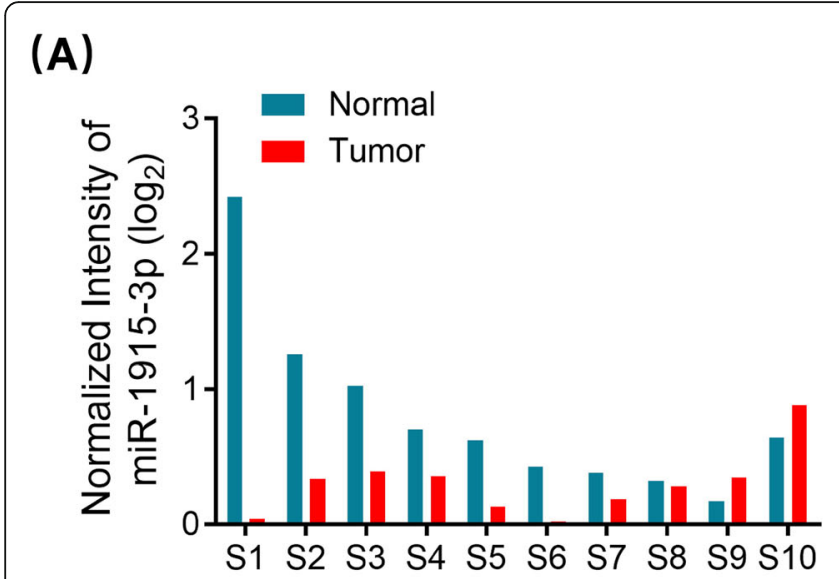

(B)

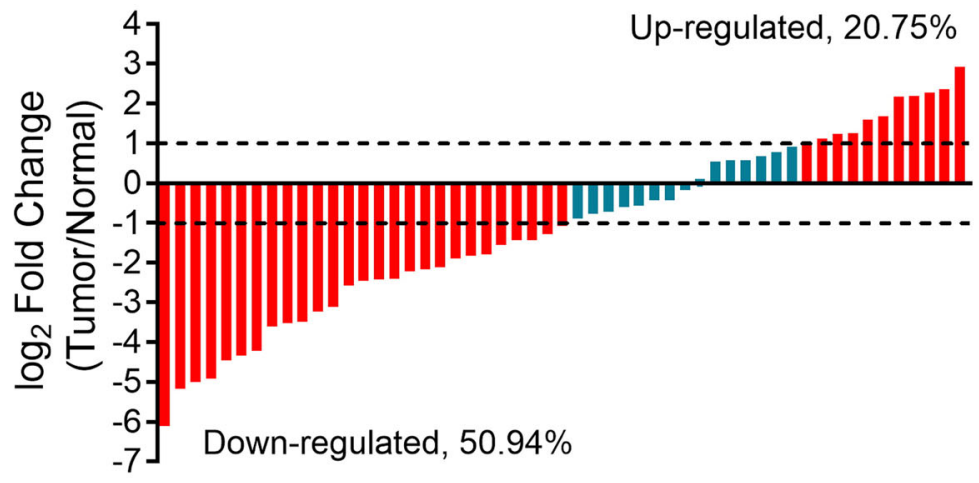

(C)
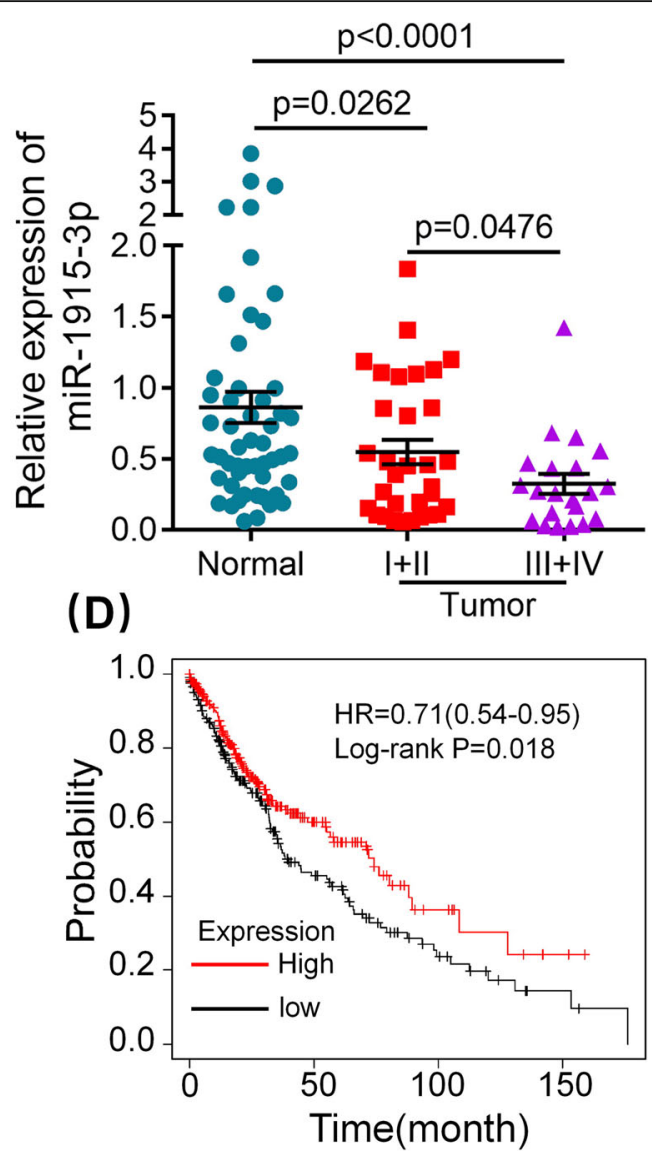

Fig. 1 miR-1915-3p is down-regulated in NSCLC tissues and inversely correlated with the progression of NSCLC. A miR-1915-3p expression in 10 matched pairs of NSCLC tissues was analyzed by miRNA microarray. B miR-1915-3p expression was frequently decreased in NSCLC tissues. A log 2 fold change more than +1 or less than -1 was considered to be significantly upregulation or downregulation, respectively. C NSCLC patients with lower miR-1915-3p expression had a more advanced TNM classification. D Kaplan-Meier survival curves of overall survival based on miR1915-3p expression in 472 NSCLC patients. The log-rank test was conducted to compare differences between two subgroups. HR: hazard ratio

level of epithelial marker E-cadherin was up-regulated and mesenchymal markers vimentin and $\mathrm{N}$-cadherin were remarkably down-regulated after miR-1915-3p overexpression (Fig. 2H). Conversely, E-cadherin was reduced and $\mathrm{N}$-cadherin/vimentin were increased after miR-1915-3p silencing (Fig. 2I). These results indicated that miR-1915-3p acted as a tumor suppressor in NSCL $\mathrm{C}$ development by inhibiting cell migration, invasion and EMT process.

\section{SET is a direct target of miR-1915-3p}

To gain further insight into how miR-1915-3p regulates cell migration and invasion, we used four bioinformatic tools (Targetscan [22], miRDB [23], miRanda [24] and miRwalk [25]) to predict miR-1915-3p target candidates. As shown in the Venn diagram (Fig. 3A), seven genes (C1RL, CHTF8, MMP24, PPP2R5D, SET, TFE3 and TSKU) were identified as possible targets of miR-1915$3 p$. Then, mRNA levels of the seven genes were detected by qRT-PCR in the miR-1915-3p overexpressed A549 cells. We found only SET expression was dramatically suppressed among these target candidates (Fig. 3B). SET had 1 potential interacting site with miR-1915-3p (Fig. 3C). Moreover, we found that SET expression was repressed both at mRNA and protein levels when transfected with the miR-1915-3p mimics (Fig. 3D, E). In contrast, miR1915-3p knockdown resulted in up-regulation of the SET expression (Fig. 3F, G), indicating that SET might be a target gene of miR-1915-3p.

To explore whether miR-1915-3p modulated SET expression directly, we conducted dual-luciferase assay using SET 3'UTR vector and found that relative luciferase activity was decreased by $78 \%$ in A549 cells and 39\% in H1975 cells when co-transfected with miR-1915-3p mimics, which was partially alleviated when the miR1915-3p binding site was deleted (Fig. 3H).

Furthermore, SET expression was examined in 38 NSCLC tissues and paired adjacent normal lung samples 
Table 2 The correlation between clinical-pathological features and miR-1915-3p expression in 73 NSCLC patients

\begin{tabular}{|c|c|c|c|c|}
\hline \multirow[b]{2}{*}{ Characteristics } & \multirow[b]{2}{*}{ NO. of cases } & \multicolumn{2}{|c|}{ miR-1915 Expression } & \multirow[b]{2}{*}{$P$ value } \\
\hline & & low & high & \\
\hline \multicolumn{5}{|l|}{ Age(y) } \\
\hline$<60$ & 34 & $22(64.71 \%)$ & $12(35.29 \%)$ & $x^{2}=0.0782$ \\
\hline$\geqq 60$ & 39 & $24(61.54 \%)$ & $15(38.46 \%)$ & $p=0.7798$ \\
\hline \multicolumn{5}{|l|}{ Gender } \\
\hline Male & 48 & $31(64.58 \%)$ & $17(35.42 \%)$ & $x^{2}=0.1482$ \\
\hline Female & 25 & $15(60.00 \%)$ & $10(40.00 \%)$ & $p=0.7003$ \\
\hline \multicolumn{5}{|l|}{ Smoking history } \\
\hline Yes & 34 & $22(64.71 \%)$ & $12(35.29 \%)$ & $x^{2}=0.0782$ \\
\hline No & 39 & $24(61.54 \%)$ & $15(38.46 \%)$ & $p=0.7798$ \\
\hline \multicolumn{5}{|l|}{ NSCLC types } \\
\hline Squamous cell carcinoma & 34 & 19 (55.88\%) & $15(44.12 \%)$ & $x^{2}=1.3887$ \\
\hline Adenocarcinoma & 39 & 27 (69.23\%) & $12(30.77 \%)$ & $p=0.2386$ \\
\hline \multicolumn{5}{|l|}{ TNM classification } \\
\hline $1+\|$ & 44 & $22(50.00 \%)$ & $22(50.00 \%)$ & $x^{2}=8.0483$ \\
\hline$I I I+I V$ & 29 & $24(82.76 \%)$ & $5(17.24 \%)$ & $p=0.0046^{\circ}$ \\
\hline \multicolumn{5}{|l|}{ EGFR } \\
\hline Del-19 & 18 & $12((66.67 \%)$ & $6(33.33 \%)$ & $x^{2}=0.2916$ \\
\hline L858R & 12 & $8(66.67 \%)$ & $4(33.33 \%)$ & $p=0.8643$ \\
\hline WT & 43 & 26 (60.47\%) & 17 (39.53\%) & \\
\hline
\end{tabular}

${ }^{a} p<0.05$ indicated statistically significant differences. Del-19 EGFR with a deletion in exon 19, L858R EGFR with L858R point mutation in exon 21, WT Wild-type EGFR. The Chi-square test was used to determine the correlation between miR-1915-3p expression and clinicopathological parameters

by qRT-PCR. Using spearman correlation analysis, we found that miR-1915-3p was negatively associated with the expression of SET (Fig. 3I). Thus, these results indicated that SET was a direct target gene of miR-1915-3p.

\section{SET is a critical mediator of miR-1915-3p effects}

To confirm whether SET inhibition recapitulated the inhibitory effect on cell migration and invasion of miR1915-3p, we knocked down endogenous SET expression by SET siRNA (siSET) in both A549 and H1975 cells. Migration and invasion assays demonstrated that cell migratory and invasive capacities were markedly reduced after SET silence (Fig. 4A, B). Western blot assay showed that SET inhibition resulted in up-regulation of $\mathrm{E}$ cadherin and down-regulation of $\mathrm{N}$-cadherin/Vimentin (Fig. 4C).

Mody et al. indicated that SET up-regulated $\mathrm{N}$ cadherin expression through JNK/Jun signaling pathway [26]. Therefore, we examined the protein levels of members of the JNK/Jun pathway after SET silencing. As shown in Fig. 4D, the level of p-JNK and p-c-Jun were decreased after SET silencing. In addition, previous studies found SET contributed to EMT by activating the nuclear factor- $\mathrm{kB}(\mathrm{NF}-\mathrm{kB})$ pathway $[27,28]$. We then examined the expression of proteins involved in NF- $\mathrm{B}$ signaling. The level of NF- $\mathrm{BB}$ activation complex pro-

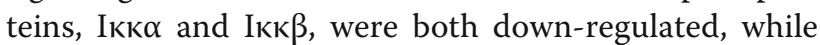
$\mathrm{I} \kappa \mathrm{B} \alpha$, as a NF- $\mathrm{B}$ inhibitor, was significantly upregulated when SET silenced (Fig. 4E). NF-кB p65 subunit, which plays important role in its transcription activity, was also remarkedly reduced in siSET group (Fig. 4E).

Together, the tumor suppressor effects of SET inhibition were similar to those of overexpression of miR1915-3p. SET inhibition attenuated EMT phenotype through JNK/Jun and NF- $\mathrm{BB}$ signaling pathway in lung cancer cells.

Next, we further examined whether restoration of SET expression was involved in miR-1915-3p-induced suppression of NSCLC cell migration and invasion. Overexpression of miR-1915-3p alone suppressed migration and invasion of NSCLC cells, and concomitant reexpression of SET could partially overturn these effects (Fig. 5A, B). Consistent with this, the restoration of SET expression noticeably abrogated the miR-1915-3p-mediated inhibition of $\mathrm{N}$-cadherin/Vimentin and induction of E-cadherin (Fig. 5C). Cells incubated with miR-1915-3p mimic alone showed a significant inhibition in JNK/Jun and NF- $\mathrm{kB}$ signaling pathway, whereas ectopic expression of SET attenuated these effects (Fig. 5D, E). 

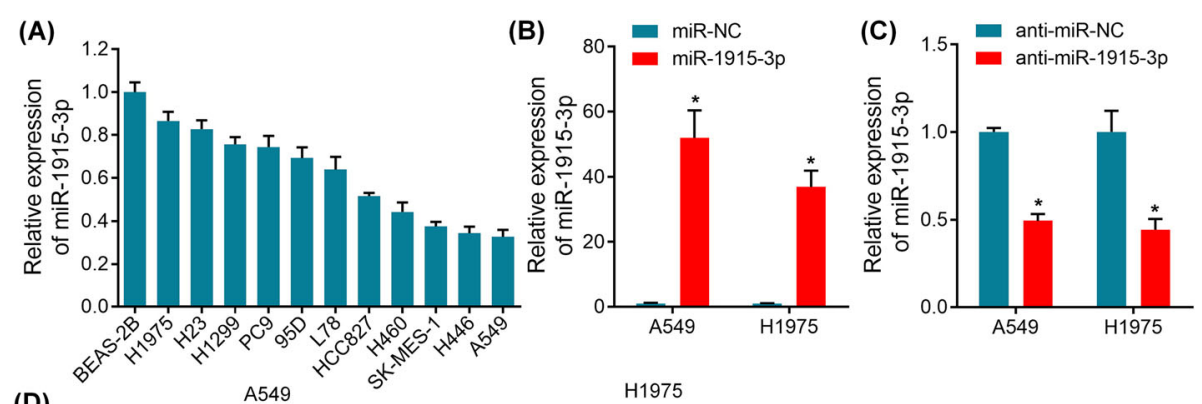

(D)
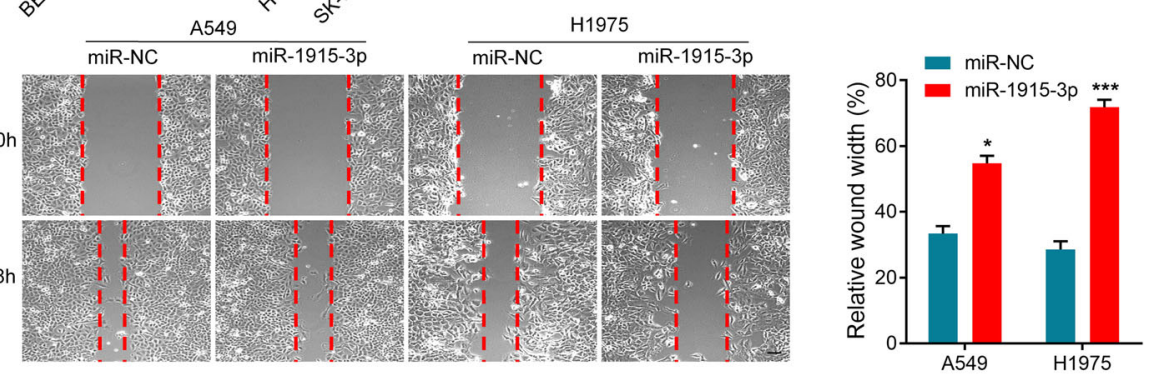

(E)
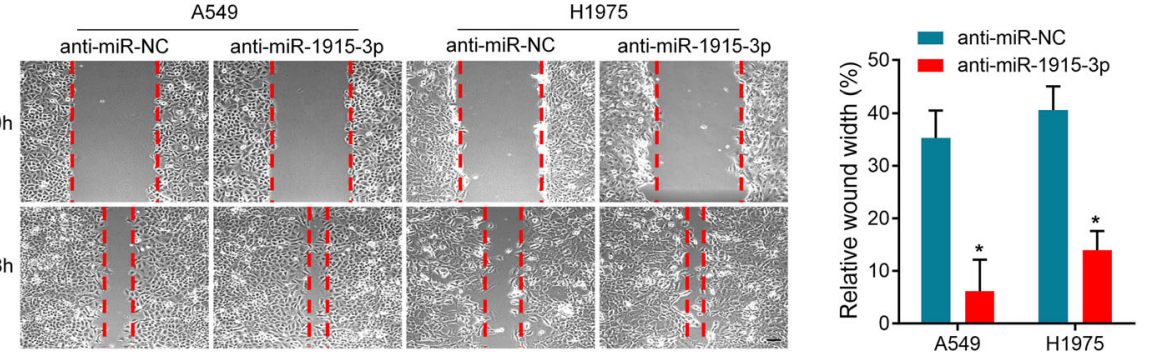

(F)
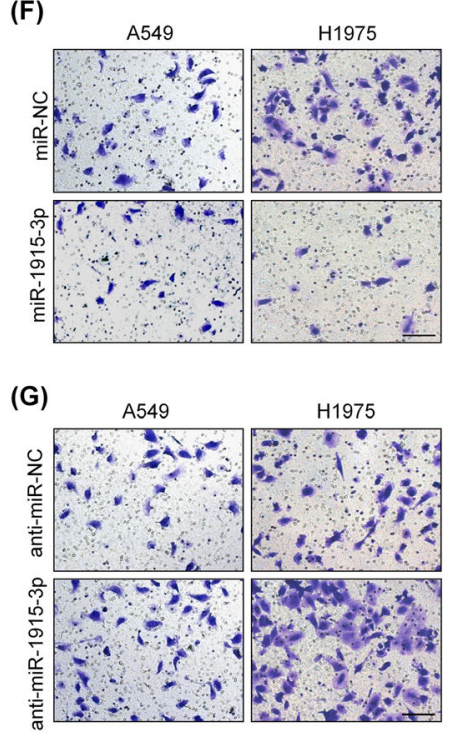
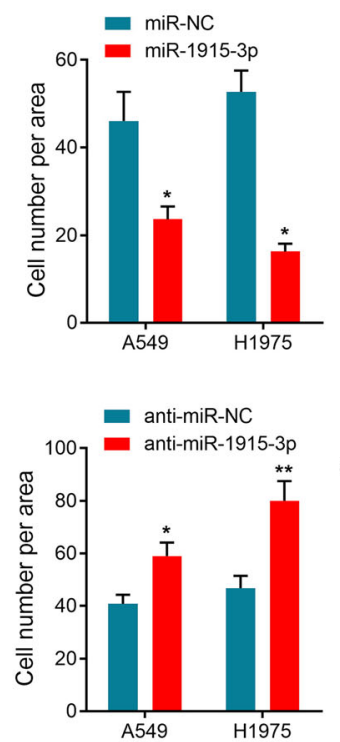

(H)
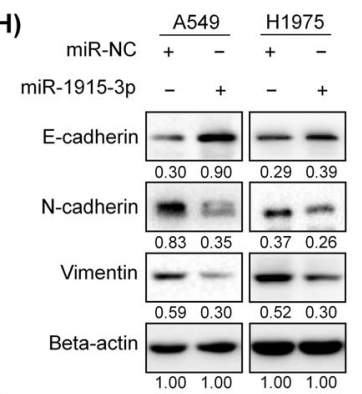

(I)
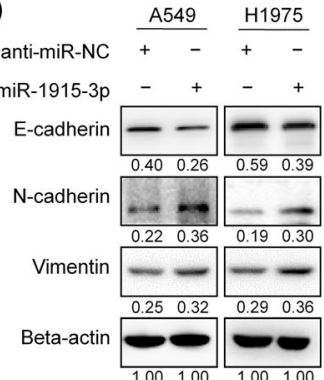

Fig. 2 miR-1915-3p suppresses the migration, invasion and EMT of NSCLC cells. A miR-1915-3p expression was analyzed by qRT-PCR in eleven lung cancer cell lines and a human normal bronchial epithelial cell line. B, C miR-1915-3p expression levels in miR-1915-3p/anti-miR-1915-3ptransfected A549 and H1975 cells. D, E Wound healing assay was carried out to determine cell migratory abilities in A549 and H1975 cells transfected with miR-NC, miR-1915-3p mimic, anti-miR-NC, or anti-miR-1915-3p. Relative wound width were presented in the histogram on the right. F, G Cell invasion assay was performed in transfected-A549 and H1975 cells as described in (D) (E). The number of invading cells per area was counted. Data represent the means \pm SEM from three independent experiments. ${ }^{*} p<0.05,{ }^{* *} p<0.01,{ }^{* * *} p<0.001$. Scale bars, $100 \mu \mathrm{m}$. (H)(I) Western blot analysis of EMT markers in miR-1915-3p/anti-miR-1915-3p-transfected A549 and H1975 cells 


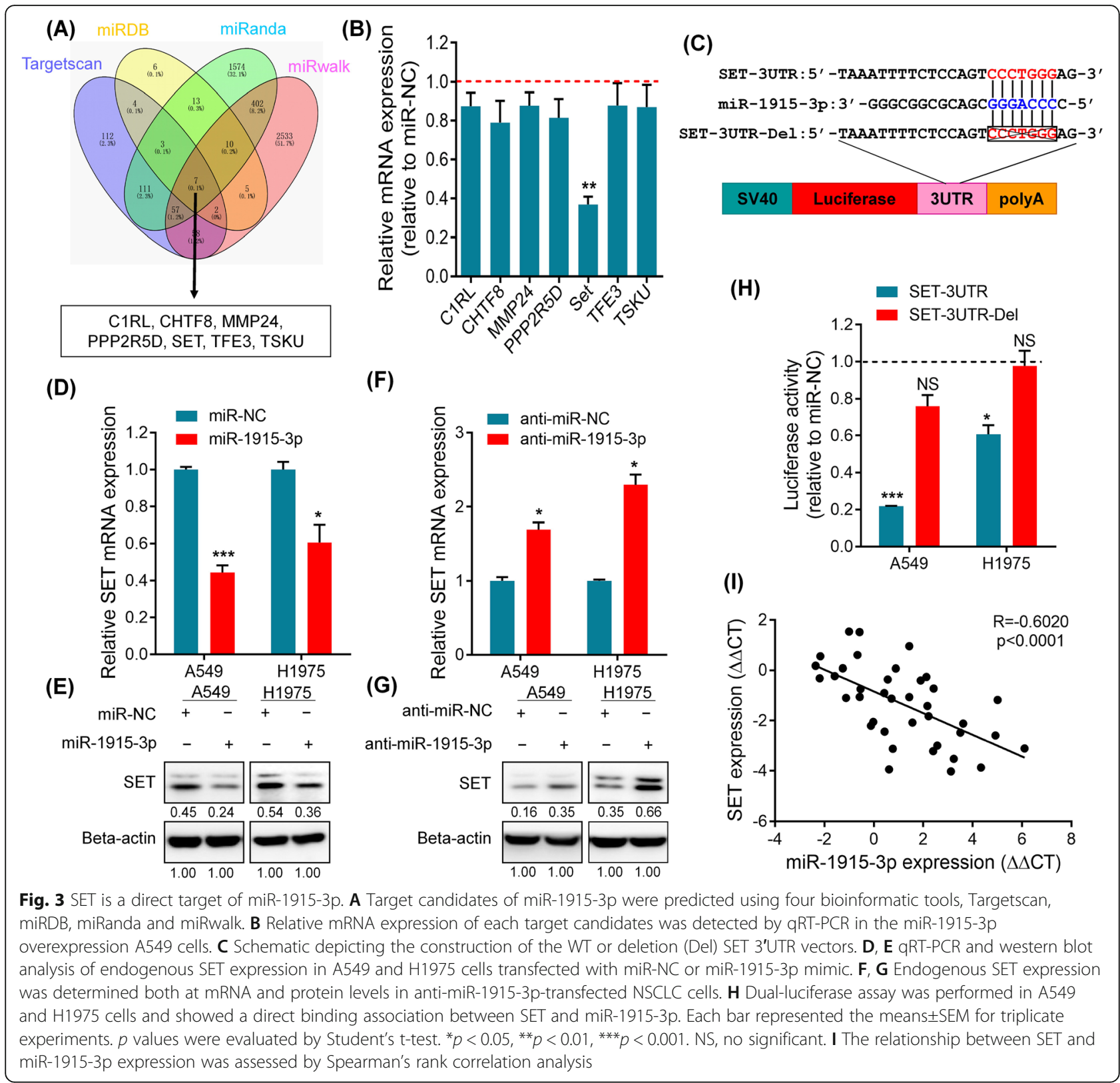

Collectively, our findings demonstrated that miR1915-3p suppressed migratory and invasive capacities of NSCLC cells partly through direct targeting of SET.

\section{SET is highly expressed in NSCLC}

To determine the expression of SET in NSCLC, the human protein atlas (https://www.proteinatlas.org/) were used to analyze SET expression at protein levels by immunohistochemistry (IHC). The IHC results showed that SET staining was strong in NSCLC specimens, but very weak in normal lung samples (Fig. 6A), suggesting that SET was highly expressed in NSCLC at protein levels. Moreover, we examined SET mRNA expression in 38 pairs NSCLC tissues and found that SET expression was significantly elevated in tumor samples (Fig. 6B). Similar results observed in GSE30219 dataset [29], NSCLC patients showed higher expression of SET as compared with normal tissues (Fig. 6C). Additionally, the correlation of SET expression with OS in GSE30219 dataset was assessed by Kaplan-Meier plotter online database. Results demonstrated that a high SET level was associated with poor prognosis (Fig. 6D).

\section{METTL3/KLF4 axis participate in cell migration and invasion by regulating miR-1915-3p}

In addition to the effects of miR-1915-3p on cell migration and invasion, we were also intrigued with the mechanism underlying the dysregulation of miR-1915-3p in 


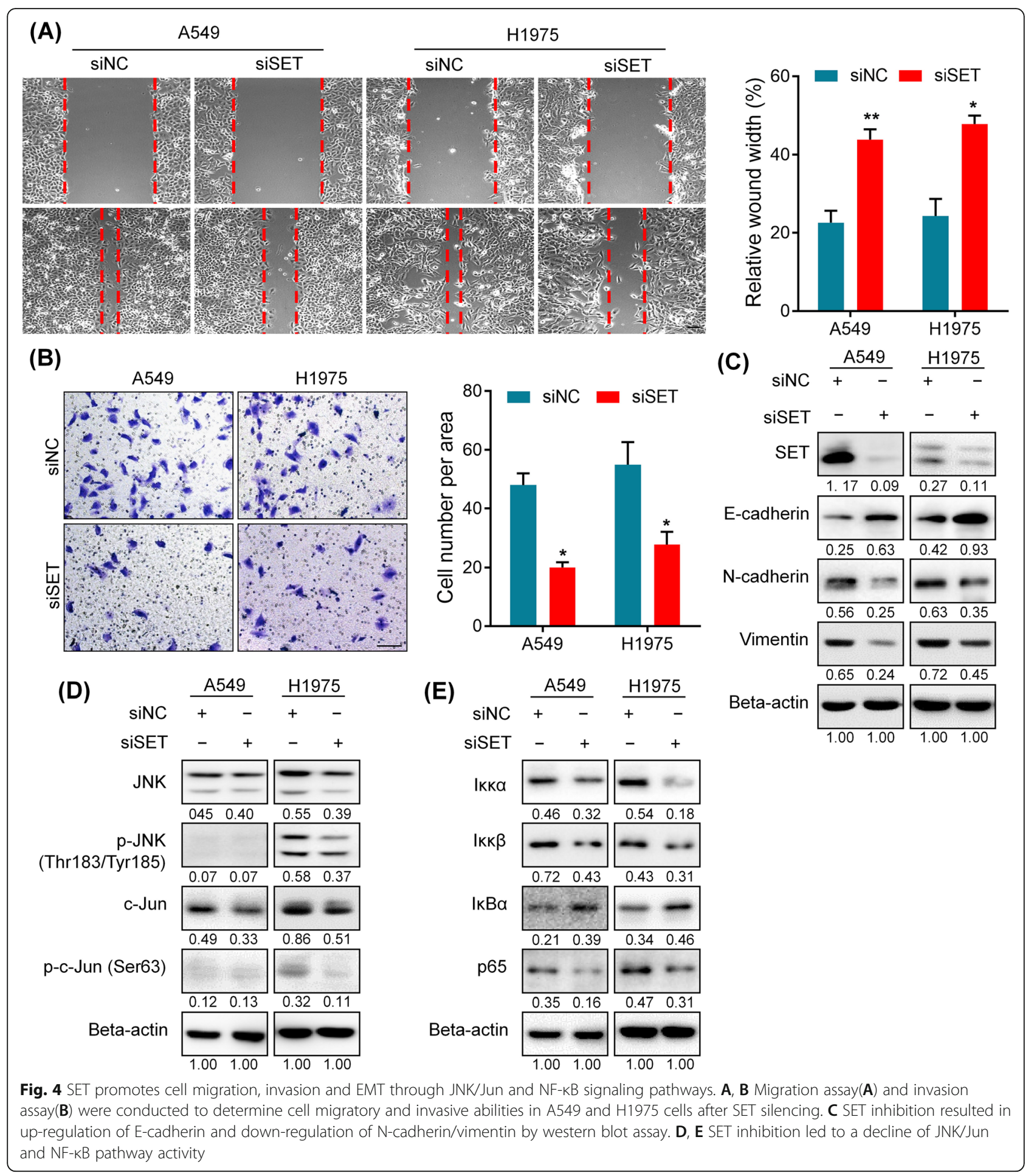

NSCLC cells. Accumulated evidence suggested that m6A modification played crucial role in the splicing process of miRNA [30], thereby leading to dysregulation of corresponding mature miRNAs. We evaluated whether METTL3, a key m6A methyltransferase, was required in the regulation of miR-1915-3p. As illustrated in Fig. 7A, METTL3 loss significantly promoted the expression of miR-1915-3p in NSCLC cells, implying that m6A modification hindered miR-1915-3p expression. m6A modification were selectively recognized by $\mathrm{m} 6 \mathrm{~A}$ "readers" which determined the fate of m6A-modified RNA and mediated m6A function [31]. Interestingly, depletion of YTHDF2, which was an important m6A reader involved in regulation of mRNA degradation [32], could 

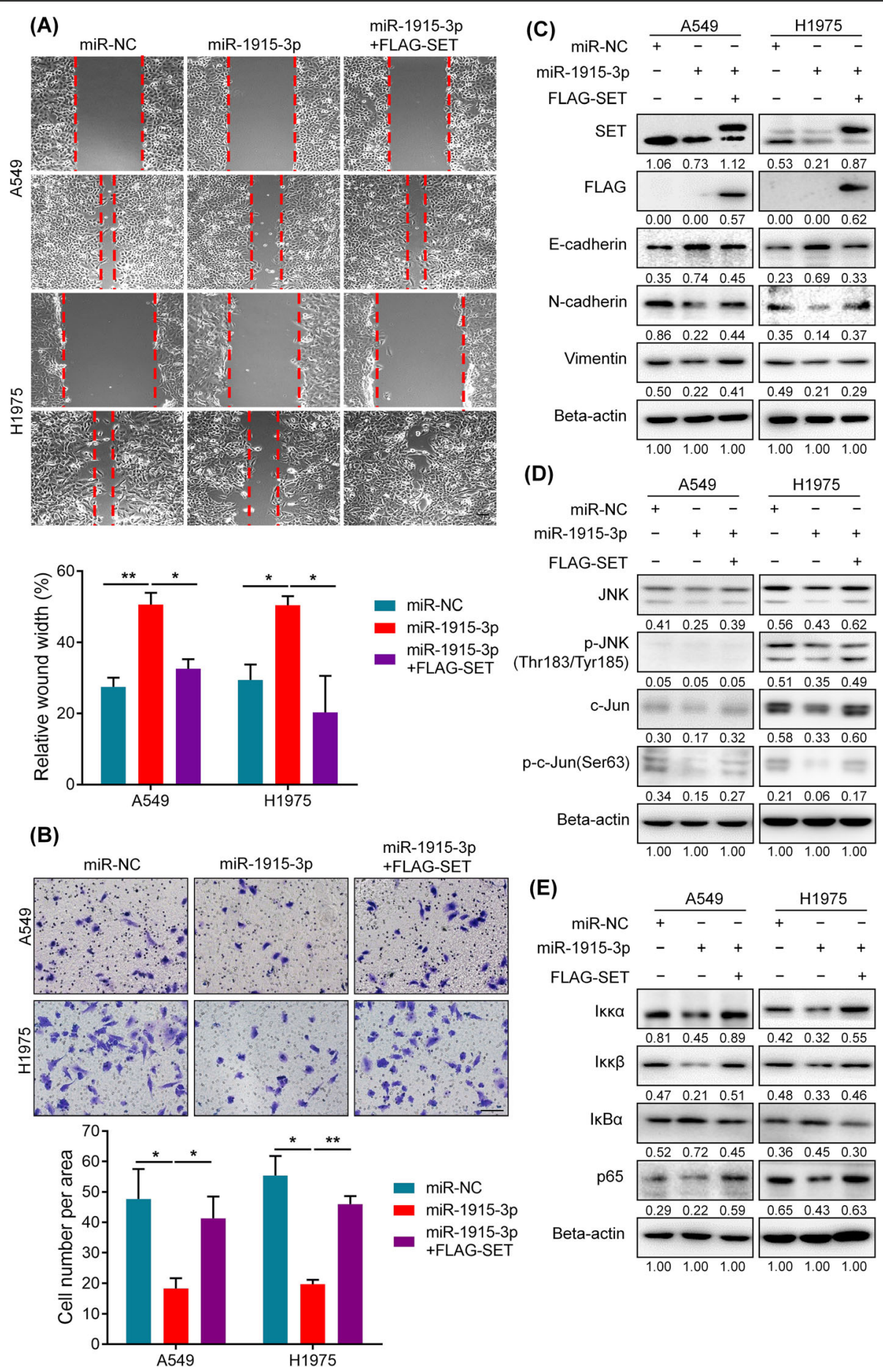

Fig. 5 SET is a critical mediator of miR-1915-3p effects. A, B miR-1915-3p suppressed migration and invasion of NSCLC cells, and re-expression of SET rescued miR-1915-3p-induced inhibitory effects. Scale bars, $100 \mu \mathrm{m}$. C The restoration of SET expression abrogated the miR-1915-3p-mediated inhibition of N-cadherin/Vimentin and induction of E-cadherin. D, E Overexpression of miR-1915-3p alone showed a significant inhibition in JNK Jun and NF-KB signaling pathway, whereas ectopic expression of SET attenuated these effects

also remarkably promote miR-1915-3p expression similar to that of the METTL3 inhibition (Fig. 7A).

To further verify whether YTHDF2 mediated the biogenesis of miR-1915-3p, we then measured the levels of pri-miR-1915 and pre-miR-1915 by qRTPCR. Our results showed that both pri- and premiR-1915 expression were up-regulated after YTHD F2 inhibition (Fig. 7B), indicating that YTHDF2 was 


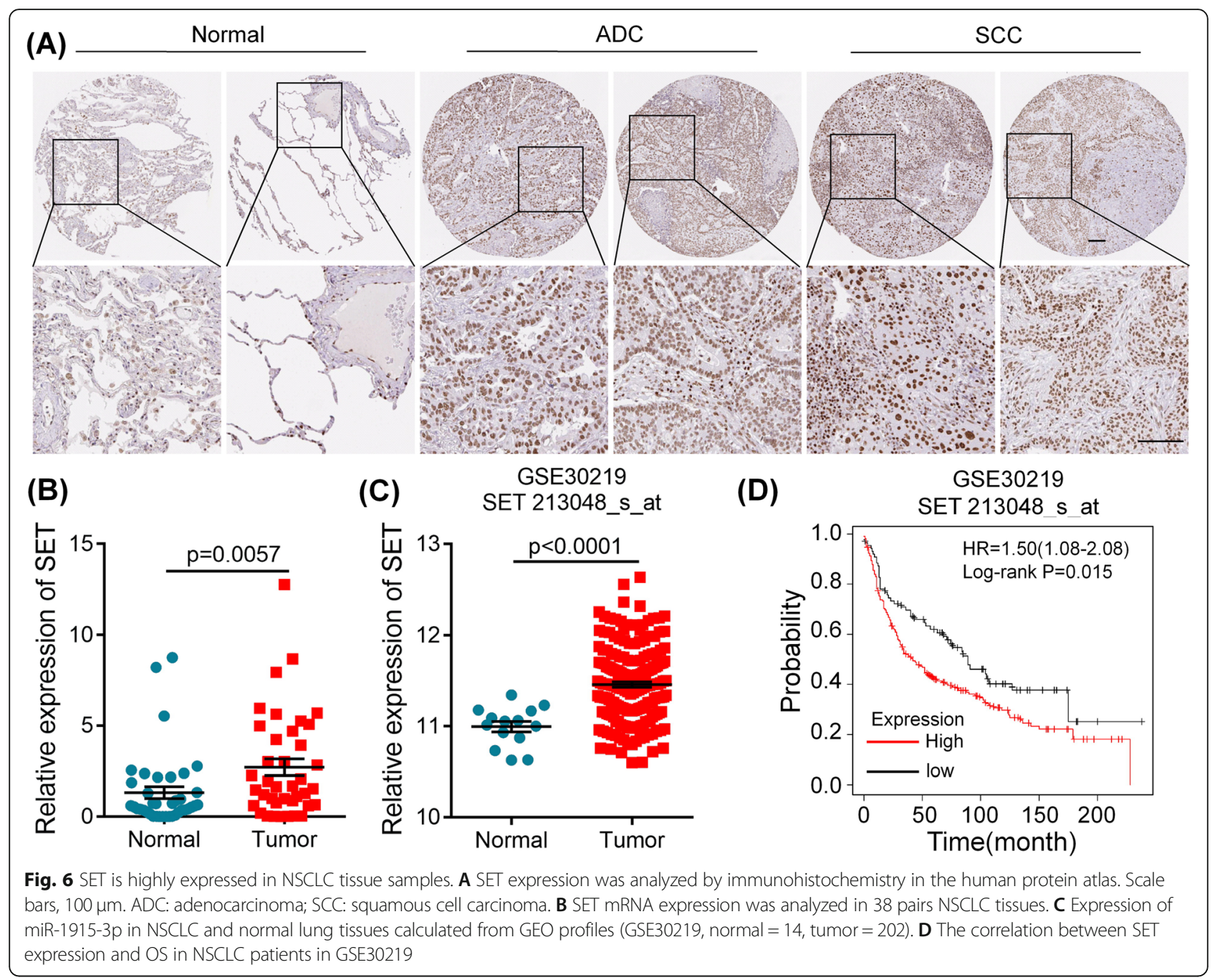

not implicated in the processing of miR-1915, but might control the regulation of miR-1915-3p at transcription levels.

As previous research showed that in bladder cancer METTL3/YTHDF2 m6A axis accelerates carcinogenesis by degrading transcription factor KLF4 [33], we also examined whether METTL3/YTHDF2 m6A axis inhibited KLF4 expression in lung cancer. We found KLF4 expression were enhanced following METTL3 or YTHDF2 knockdown in lung cancer cells (Fig. 7C). In addition, the expression level of miR-1915-3p was decreased in KLF4 knockdown cells, while the level of miR-1915-3p was increased in KLF4 overexpression cells (Fig. 7D). In order to identify whether KLF4 directly promote miR1915-3p expression by binding to miR-1915 promoter, we analyzed the genome sequence around the transcription start site (TSS) of miR-1915, noting the presence of two putative KLF4-binding sequences (CACCC) (Fig. 7E). We then constructed miR-1915 promoter reporter and deletion mutants, found that miR-1915 promoter had high transcription activity, and KLF4-binding site deletion led to a decline of transcription activity (Fig. 7F). Promoter luciferase assays indicated KLF4 increased the transcription activity of miR-1915 promoter, and deleting both KLF4-binding sites diminished KLF4-mediated reporter induction (Fig. 7G). CHIP assays using KLF4 antibody showed enrichment of miR-1915 promoter in A549 and H1975 cells, whereas negative control (IgG and Ab-groups) did not (Fig. 7H), demonstrating that KLF4 directly bound to the miR-1915 promoter in lung cancer cells and transcriptionally regulated miR-1915 expression. These results indicated that METTL3/YTHDF2 m6A axis regulated miR-1915-3p expression through transcription factor KLF4.

Next, we further examined the effects of METTL3/ KLF4 axis on cell migration and invasion. We found that METTL3 silencing or KLF4 overexpression could 


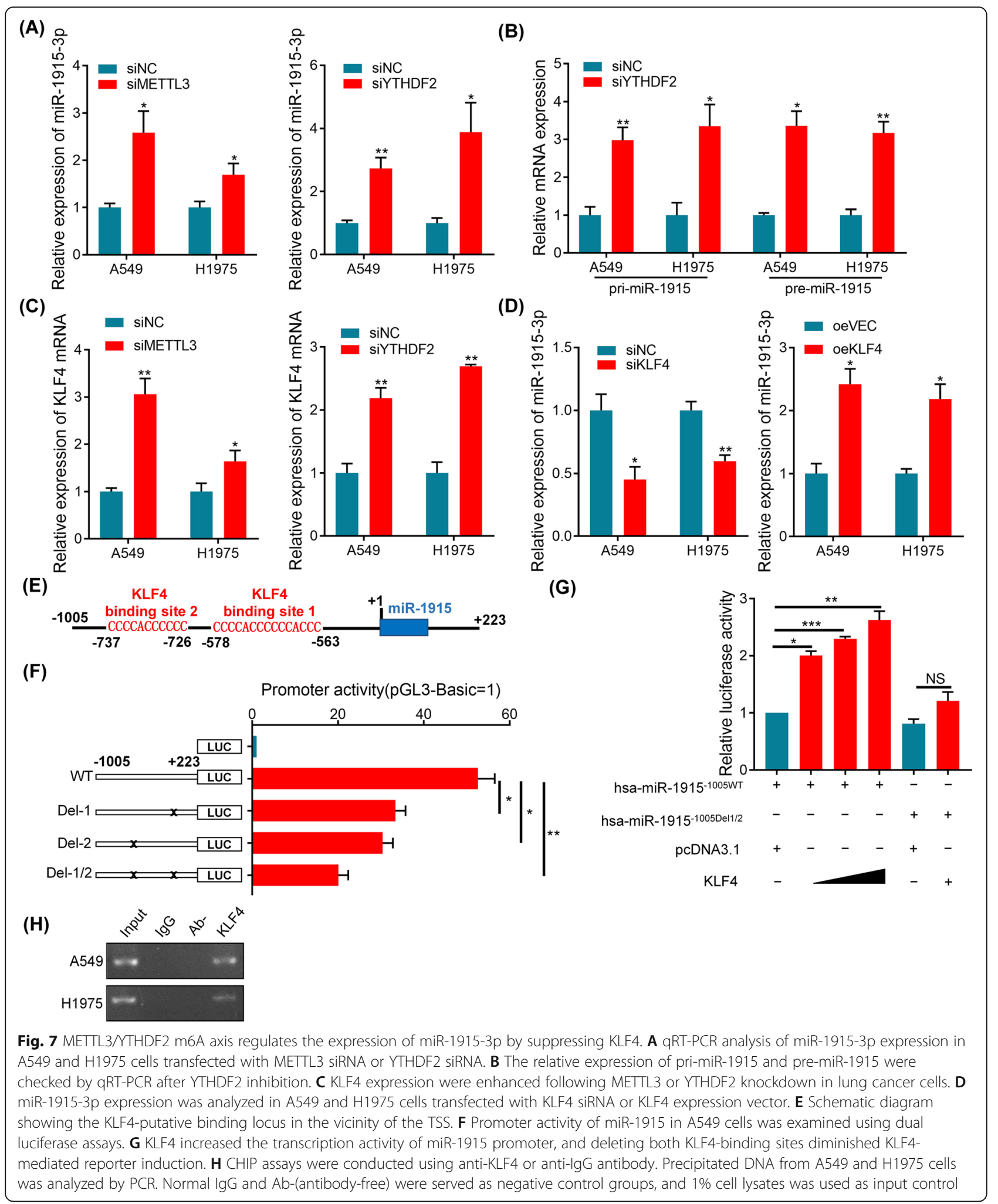

suppress cell migration and invasion, and concomitant miR-1915-3p inhibition partially overturn these effects (Fig. 8A, B). Consistent with this, METTL3 silencing or
KLF4 overexpression inhibited EMT, JNK/Jun and NF$\kappa B$ signaling pathways, whereas miR-1915-3p inhibition attenuated these effects (Fig. 8C, D, E). 


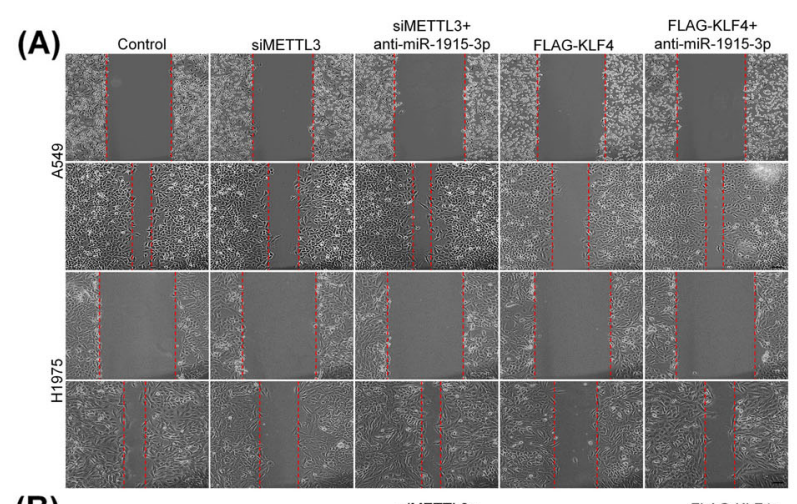

(B)

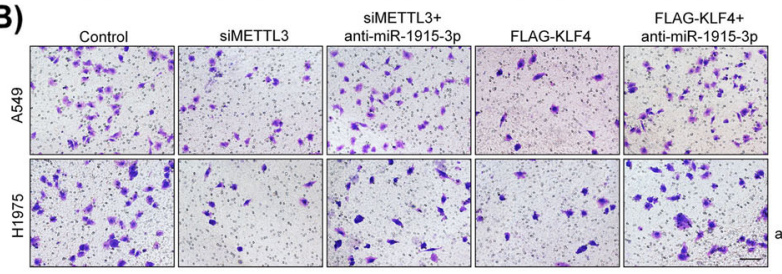

(C)

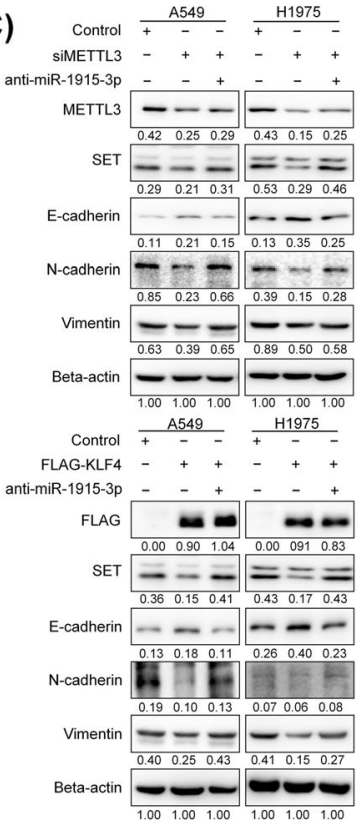

(D)

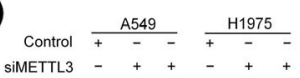

anti-miR-1915-3p

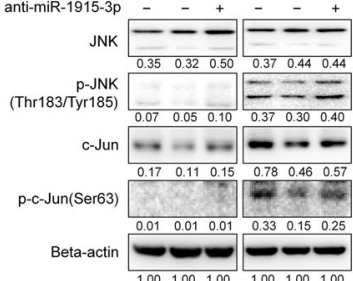

$\mathrm{A} 549$
$+-\mathbf{H}$

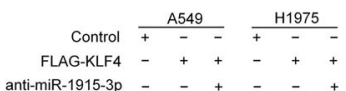

anti-miR-1915-3p

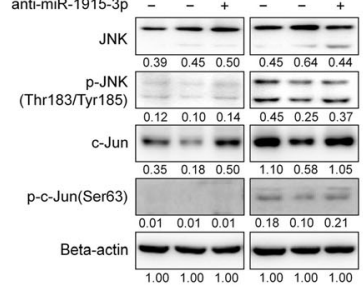

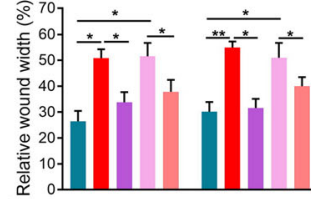

Control $+\ldots-\cdots+\cdots$

SiMETTL3 - + + - - + + -

anti-miR-1915-3p - - + + + - +
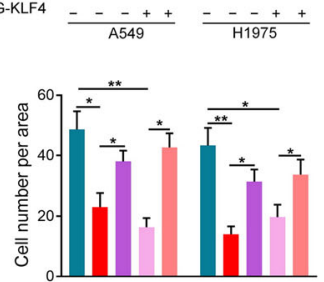

Control + -

SiMETTL3 - + + - - + + -

FLAG-KLF 4

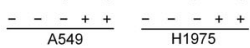

(E)

Control $\frac{\text { A549 }}{+-\frac{\mathrm{H} 1975}{+-}}$

SiMETTL3 -+
S anti-miR-1915-3p

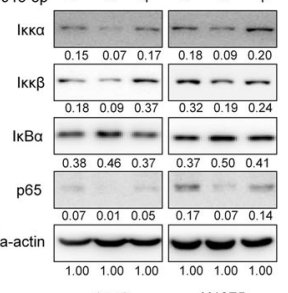

Control $\frac{\mathrm{A} 549}{+-\frac{\mathrm{H} 1975}{+-}}$

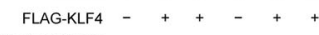

nti-miR-1915-3p

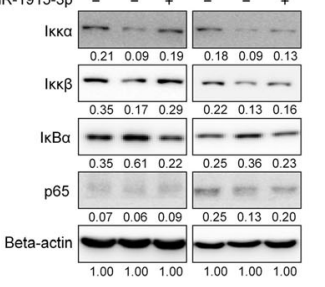

Fig. 8 METTL3/KLF4 axis participate in cell migration and invasion by regulating miR-1915-3p. A, B Migration assay(A) and invasion assay(B) were conducted to determine cell migratory and invasive abilities in A549 and H1975 cells after METTL3 silencing or KLF4 overexpression. Scale bars, $100 \mu \mathrm{m}$. C, D, E Western blot analysis for related proteins that were involved in EMT, JNK/Jun and NF-KB pathways

\section{Discussion}

Lung cancer is the second most commonly diagnosed cancer with high fatality in the world. Increasing evidences have shown that miRNAs play significant roles in carcinogenesis and development of NSCLC.

In this report, our results revealed that miR-1915-3p was markedly repressed in NSCLC cell lines and tissues and that down-regulation of miR-1915-3p was inversely associated with clinical TNM stage and overall survive, suggesting that miR-1915-3p may function as a tumor suppressor in NSCLC development. Similarly, previous results showed that the relative expression of miR-1915$3 p$ was down-regulated in gastric cancer and modulated the development of gastric cancer through the repression of RAGE and BCL-2 [19, 34]. Down-regulation of miR1915-3p was responsible for multidrug resistance in colorectal cancer, and re-establishing miR-1915-3p could restore the chemosensitivity depend on suppression of BCL-2 and NFIX expression [17, 35]. Jin et al. demonstrated that there were low levels of miR-1915-3p in breast cancer, which inhibited H3K4 methylation, proliferation and migration by targeting SETD1A [36]. Consistent with 
our findings, these results indicated that miR-1915-3p functioned as a tumor suppressor and blocked tumor progression by regulating several signaling pathways. However, opposite results are also obtained by some previous researches. Wan and colleagues showed that miR1915-3p was upregulated by p53-dependent pathway under oxidative stress in hepatocellular carcinoma [37]. In thyroid carcinomas, high expression levels of miR-1915$3 \mathrm{p}$ associated with infiltrative growth of follicular variant of papillary thyroid carcinomas [38]. Guo et al. reported that miR-1915-3p was markedly higher in breast cancer patient's serum and associated with lymph node metastasis. Ectopic expression of miR-1915-3p enhanced cell proliferative and migratory capacities by repressing of DUSP3 and activation of ERK1/2 signaling [39]. In addition, miR1915-3p could impair etoposide-induced apoptosis, thereby increasing chemoresistance via downregulating DRG2 and PBX2 in lung cancer cell lines (NCI-H441 and NCI-H1650) [40]. These divergent results might be due to the differences in various cancers, cell types or tumor heterogeneity. In the present study, we found that miR-1915$3 p$ inhibited migration, invasion and EMT of NSCLC cells, acting as a tumor suppressor. Through bioinformatic analysis, SET was predicted as a potential target gene of miR-1915-3p. Then the dual-luciferase reporter assay confirmed that miR-1915-3p could directly interact with SET 3'UTR, thereby depressing the expression of SET in NSCLC cells. Therefore, our results demonstrated that miR-1915-3p enforced its anti-oncogenic role through directly targeting SET, and restoration of miR-1915-3p in NSCLC may hold therapeutic promise.

SET, an endogenous inhibitor of PP2A, plays a pivotal oncogenic role in tumorigenesis [41]. SET has been identified as an oncogene in multiple types of cancers, such as hepatocellular carcinoma [42], pancreatic cancer [26], glioblastoma multiforme [43] and lung cancer [44, 45]. In the current study, we confirmed that SET was substantially increased in NSCLC specimens and associated with worse clinical outcome, which was consistent with previous studies [44, 45]. In addition, previous studies showed that SET could promote EMT and metastasis by modulating several signaling pathways. Liu et al. concluded that SET could depress the expression of NDRG1 through PP2A/c-MYC pathway, resulting in activation of EMT process, whereas blockade of SET by FTY720 (fingolimod) could inhibit EMT and restore the chemosensitivity in A549 lung cancer cells [27]. NDRG1 upregulated E-cadherin via NF- $\mathrm{KB}$ signaling pathway [28]. Mody et al. suggested that SET mediated EMT transition by promoting N-cadherin expression through Rac1/JNK/ c-Jun signaling pathway in pancreatic cancer [26]. Herein, our findings indicated that knockdown of SET led to repression of migration and invasion, upregulation of epithelial marker (E-cadherin), and downregulation of mesenchymal markers (N-cadherin/Vimentin) in NSCL C cells, which recapitulated miR-1915-3p-induced inhibitory effects on cell migration, invasion and EMT process through JNK/c-Jun and NF- $\mathrm{KB}$ signaling pathways. Furthermore, we verified that miR-1915-3p controlled cancer migration, invasion and EMT process through directly downregulating SET in lung cancer cells.

M6A marks have been identified as a key regulator of miRNA biogenesis by suppressing the binding of DGCR8 to pri-miRNAs [46]. Aberrant expression of METTL3 may be significantly responsible for the alteration of miRNAs. It has been reported that METTL3 modulated the pri-miR221/222 and pri-miR1246 process in a DGCR8-dependent manner [47, 48]. In addition, previous study indicated that METTL3 could facilitate the cleaving of miR-143 precursor to form mature miRNA in lung cancer [49]. Meanwhile, Alarcon et al. have shown that m6A "reader" protein HNRNPA2B1 can directly bind to m6A marks of primary miRNA transcripts to modulate miRNA biogenesis [50]. Our present study pointed out that METTL3 loss significantly promoted the expression of miR-1915-3p, whereas HNRN PA2B1 had no effect on the expression of miR-1915-3p (data not show). However, depletion of m6A "reader" protein YTHDF2 could promote miR-1915-3p expression and result in accumulation of pri/pre-miR-1915, indicating that YTHDF2 was not involved in the processing of miR-1915, and might control miR-1915-3p expression through transcriptional regulation. Xie and colleagues have determined that the METTL3/YTHDF2 m6A axis directly degraded the mRNA of the transcription factor KLF4, contributing to the progression of bladder cancer [33]. Subsequently, we validated that METTL3/YTHDF2 m6A axis also repressed KLF4 expression in lung cancer. Furthermore, KLF4 directly bound to the miR-1915 promoter and transcriptionally regulated miR-1915 expression in lung cancer cells. METTL3 silencing or KLF4 overexpression could suppress cell migration and invasion, and concomitant miR1915-3p inhibition partially overturn these effects. These results indicated that METTL3/KLF4 axis participated in cell migration and invasion by regulating miR-1915-3p.

\section{Conclusion}

Collectively, we identified that miR-1915-3p, as a crucial tumor suppressor, was significantly down-regulated in NSCLC, and inversely associated with clinical TNM stage and overall survival. Functional studies indicated that miR-1915-3p suppressed cancer migration, invasion and EMT of NSCLC via directly targeting oncogene SET. In addition, the expression of miR-1915-3p was regulated by METTL3/YTHDF2 m6A axis through transcription factor KLF4. These findings that the 
dysregulation of miR-1915-3p was involved in NSCLC metastasis indicate that miR-1915-3p may have an antimetastatic therapeutic potential for lung cancer treatment.

\section{Abbreviations}

miRNA: microRNAs; NSCLC: Non-small-cell lung cancer; qRT-PCR: Quantitative real-time PCR; CHIP: Chromatin immunoprecipitation; EMT: Epithelialmesenchymal transition; PROM1: Prominin 1; PAX2: Paired box 2

\section{Acknowledgements}

Not applicable.

\section{Authors' contributions}

PHL, LXB and ZQH conceived and designed the study. PZH, GFJ, WMM, MFR $Z L L, L Y, D X X, Z X W, S Y, F Y G$, and LMJ did the experiments. All authors have read and approved the final version of this manuscript.

\section{Funding}

This work was supported by grants from the Tianjin Natural Science Foundation (No.17JCQNJC11700, to Hongli Pan; No.18JCYBJC92100, to Xuebing Li; No.18JCQNJC12700, to Yi Shao), National Natural Science Foundation of China (No.81302002, to Xuebing Li), the Key Project of Cancer Foundation of China (No. CFC2020kyxm003, to Xuebing Li), Foundation of Tianjin Medical University General Hospital (No. ZYYFY2016013, to Hongli Pan and No. ZYYFY2019022, to Fanrong Meng), the Introduction of talents and doctoral initiation fund of Tianjin Medical University Cancer Institute and Hospital (grant number B1917), the Startup Project of Tianjin Lung Cancer Institute (No. TJLCZJ2021-06, to Hongli Pan) and the General Project of Tianjin Lung Cancer Institute (No. TJLCMS2021-03, to Xuebing Li).

\section{Availability of data and materials}

The datasets used and/or analyzed during the current study are available from the corresponding author on reasonable request.

\section{Declarations}

\section{Ethics approval and consent to participate}

The human tissue samples were used according to the guidelines and regulations of Tianjin Medical University General Hospital. This study was approved by the ethics committee of Tianjin Medical University General Hospital and written informed consent was obtained from all patients.

\section{Consent for publication}

Not applicable.

\section{Competing interests}

The authors declare no conflicts of interest.

\section{Author details}

${ }^{1}$ Tianjin Key Laboratory of Lung Cancer Metastasis and Tumor Microenvironment, Tianjin Lung Cancer Institute, Tianjin Medical University General Hospital, Tianjin, China. ${ }^{2}$ Tianjin Prenatal Diagnostic Center, Obstetrics and Gynecology Department, Tianjin Medical University General Hospital, Tianjin, China. ${ }^{3}$ Sichuan Lung Cancer Center, West China Hospital, Sichuan University, Chengdu, China. ${ }^{4}$ Department of Lung Cancer Surgery, Tianjin Medical University General Hospital, Tianjin, China. ${ }^{5}$ Department of Thoracic Surgery, Yizheng People's Hospital, Yangzhou, Jiangsu Province, China. ${ }^{6}$ Department of Oncology, Tianjin Medical University General Hospital, Tianjin, China. ${ }^{7}$ The State Key Laboratory of Medicinal Chemical Biology, College of Pharmacy, Nankai University, Tianjin, China.

Received: 20 May 2021 Accepted: 2 November 2021 Published online: 13 November 2021

\section{References}

1. Sung H, Ferlay J, Siegel RL, et al. Global cancer statistics 2020: GLOBOCAN estimates of incidence and mortality worldwide for 36 cancers in 185 countries. CA Cancer J Clin. 2021;0:1-41.
2. Zappa C, Mousa SA. Non-small cell lung cancer: current treatment and future advances. Transl Lung Cancer Res. 2016;5(3):288-300. https://doi. org/10.21037/t|cr.2016.06.07.

3. Wang X, Adjei AA. Lung cancer and metastasis: new opportunities and challenges. Cancer Metastasis Rev. 2015;34(2):169-71. https://doi.org/10.1 007/s10555-015-9562-4.

4. Siegel RL, Miller KD, Jemal A. Cancer statistics, 2020. CA Cancer J Clin. 2020; 70(1):7-30. https://doi.org/10.3322/caac.21590.

5. Lee RC, Feinbaum RL, Ambros V. The C.elegans heterochronic gene lin-4 encodes small RNAs with antisense complementarity to lin-14. Cell. 1993; 75(5):843-54. https://doi.org/10.1016/0092-8674(93)90529-Y.

6. Moretti F, Thermann R, Hentze MW. Mechanism of translational regulation by miR-2 from sites in the $5^{\prime}$ untranslated region or the open reading frame. RNA. 2010;16(12):2493-502. https://doi.org/10.1261/ rna.2384610.

7. Forman J, Legesse-Miller A, Coller HA. A search for conserved sequences in coding regions reveals that the let-7 microRNA targets dicer within its coding sequence. Proc Natl Acad Sci. 2008;105(39):14879-84. https://doi. org/10.1073/pnas.0803230105.

8. Chou J, Lin JH, Brenot A, Kim JW, Provot S, Werb Z. GATA3 suppresses metastasis and modulates the tumor microenvironment by regulating microRNA-29b expression. Nat Cell Biol. 2013;15(2):201-13. https://doi.org/1 $0.1038 /$ ncb2672

9. Yin Z, Wang W, Qu G, Wang L, Wang X, Pan Q. MiRNA-96-5p impacts the progression of breast cancer through targeting FOXO3. Thorac Cancer. 2020;11(4):956-63. https://doi.org/10.1111/1759-7714.13348.

10. Schnepp RW, Khurana P, Attiyeh EF, Raman P, Chodosh SE, Oldridge DA et al. A LIN28B-RAN-AURKA signaling network promotes neuroblastoma tumorigenesis. Cancer Cell. 2015;28(5):599-609. https://doi.org/10.1016/j. ccell.2015.09.012.

11. Hatley ME, Patric DM, Garcia MR, et al. Modulation of K-Ras-dependent lung tumorigenesis by MicroRNA-21. Cancer Cell. 2010;18(3):282-93. https://doi. org/10.1016/j.ccr.2010.08.013

12. Misso G, Di Martino MT, De Rosa G, et al. Mir-34: a new weapon against cancer? Mol Ther Nucleic Acids. 2014;3(9):e195. https:/doi.org/10.1038/ mtna.2014.47.

13. Cortez MA, Ivan C, Valdecanas D, et al. PDL1 regulation by p53 via miR-34. J Natl Cancer Inst. 2015:108(1):djv303.

14. Rupaimoole R, Slack FJ. MicroRNA therapeutics: towards a new era for management of cancer and other diseases. Nat Rev Drug Discov. 2017;16(3): 203-22. https://doi.org/10.1038/nrd.2016.246.

15. Yu AM, Jian C, Yu AH, Tu MJ. RNA therapy: are we using the right molecules? Pharmacol Ther. 2019;196:91-104. https://doi.org/10.1016/j.pha rmthera.2018.11.011.

16. Pan HL, Wen Z, Huang Y, et al. Down-regulation of microRNA-144 in air pollution-related lung cancer. Sci Rep. 2015;5(1):14331. https://doi.org/10.103 8/srep14331.

17. Xu K, Liang X, Cui DL, Wu YX, Shi W, Liu J. miR-1915 inhibits BCl-2 to modulate multidrug resistance by increasing drug-sensitivity in human colorectal carcinoma cells. Mol Carcinog. 2013;52(1):70-8. https://doi.org/1 $0.1002 / \mathrm{mc} .21832$

18. Nakazawa K, Dashzeveg N, Yoshida K. Tumor suppressor p53 induces miR-1915 processing to inhibit $\mathrm{BCl}-2$ in the apoptotic response to DNA damage. FEBS J. 2014;201(13):2937-44. https://doi.org/10.1111/febs.12 831.

19. Cui H, Han W, Hou L, et al. miR-1915-3p inhibits Bcl-2 expression in the development of gastric cancer. Biosci Rep. 2019;39(5): BSR20182321

20. Sallustio F, Serino G, Costantino V, et al. miR-1915 and miR-1225-5p regulate the expression of CD133, PAX2 and TLR2 in adult renal progenitor cells. PLoS One. 2013:8(7):e68296.

21. Gyorffy B, Surowiak P, Budczies J, Lanczky A. Online survival analysis software to assess the prognostic value of biomarkers using transcriptomic data in non-small-cell lung cancer. PLoS One. 2013;8(12):e82241. https://doi. org/10.1371/journal.pone.0082241.

22. Agarwal V, Bell GW, Nam JW, Bartel DP. Predicting effective microRNA target sites in mammalian mRNAs. eLife. 2015;4:e05005. https://doi.org/10.7554/ eLife.05005.

23. Liu W, Wang X. Prediction of functional microRNA targets by integrative modeling of microRNA binding and target expression data. Genome Biol. 2019;20(1):18. https://doi.org/10.1186/s13059-019-1629-z. 
24. Betel D, Wilson M, Gabow A, Marks DS, Sander C. The microRNA.org resource: targets and expression. Nucleic Acids Res. 2008;36(Database) D149-53. https://doi.org/10.1093/nar/gkm995.

25. Sticht C, De La Torre C, Parveen A, Gretz N. miRWalk: An online resource for prediction of microRNA binding sites. PLoS One. 2018;13(10):e0206239.

26. Mody HR, Hung SW, Naidu K, Lee H, Gilbert CA, Hoang TT, et al. SET contributes to the epithelial-mesenchymal transition of pancreatic cancer. Oncotarget. 2017;8(40):67966-79. https://doi.org/10.18632/oncotarget.19067.

27. Liu H, Gu YX, Yin J, Zheng G, Wang C, Zhang Z, et al. SET-mediated NDRG1 inhibition is involved in acquisition of epithelial-to-mesenchymal transition phenotype and cisplatin resistance in human lung cancer cell. Cell Signal. 2014;26(12):2710-20. https://doi.org/10.1016/j.cellsig.2014.08.010.

28. Menezes VS, Fouani L, Huang LHM, et al. The metastasis suppressor, NDRG1, attenuates oncogenic TGF- $\beta$ and NF-KB signaling to enhance membrane Ecadherin expression in pancreatic cancer cells. Carcinogenesis. 2019;40(6): 805-18. https://doi.org/10.1093/carcin/bgy178.

29. Rousseaux S, Debernardi A, Jacquiau B, et al. Ectopic activation of germline and placental genes identifies aggressive metastasis-prone lung cancers. Sci Transl Med. 2013;5(186):186ra66.

30. Chen Y, Lin Y, Shu Y, He J, Gao W. Interaction between N6-methyladenosine (m6A) modification and noncoding RNAs in cancer. Mol Cancer. 2020;19(1): 94. https://doi.org/10.1186/s12943-020-01207-4.

31. He L, Li H, Wu A, Peng Y, Shu G, Yin G. Functions of N6-methyladenosine and its role in cancer. Mol Cancer. 2019;18(1):176. https://doi.org/10.1186/ s12943-019-1109-9.

32. Wang X, Lu ZK, Gomez A, Hon GC, Yue Y, Han D, et al. m6A-dependent regulation of messenger RNA stability. Nature. 2014;505(7481):117-20. https://doi.org/10.1038/nature12730.

33. Xie H, Li JF, Ying $Y$, Yan H, Jin K, Ma X, et al. METTL3/YTHDF2 m6A axis promotes tumorigenesis by degrading SETD7 and KLF4 mRNAs in bladder cancer. J Cell Mol Med. 2020;24(7):4097-104. https://doi.org/10.1111/jcmm.1 5063.

34. Xu XC, Zhang W, Li CX, Gao H, Pei Q, Cao BW, et al. Up-regulation of miR1915 inhibits proliferation, invasion, and migration of helicobacter pyloriinfected gastric cancer cells via targeting RAGE. Yonsei Med J. 2019;60(1): 38-47. https://doi.org/10.3349/ymj.2019.60.1.38.

35. Hu J, Cai G, Xu Y, Cai S. The plasma microRNA miR-1914* and -1915 suppresses chemoresistant in colorectal cancer patients by down-regulating NFIX. Curr Mol Med. 2016;16(1):70-82. https://doi.org/10.2174/156652401 6666151222144656.

36. Jin ML, Kim YM, Jin HL, et al. Aberrant expression of SETD1A promotes survival and migration of estrogen receptor a-positive breast cancer cells. Int J Cancer. 2018;143(11):2871-83. https://doi.org/10.1002/ijc.31853.

37. Wan $Y$, Cui $R$, Gu J, Zhang $X$, Xiang $X$, Liu C, et al. Identification of four oxidative stress-responsive microRNAs, miR-34a-5p, miR-1915-3p, miR-638, and miR-150-3p, in hepatocellular carcinoma. Oxidative Med Cell Longev. 2017;5189138:1-12. https://doi.org/10.1155/2017/5189138.

38. Borrelli N, Denaro M, Ugolini C, Poma AM, Miccoli M, Vitti $P$, et al. miRNA expression profiling of 'noninvasive follicular thyroid neoplasms with papillary-like nuclear features' compared with adenomas and infiltrative follicular variants of papillary thyroid carcinomas. Mod Pathol. 2017;30(1):39_ 51. https://doi.org/10.1038/modpathol.2016.157.

39. Guo J, Liu C, Wang W, Liu Y, He H, Chen C, et al. Identification of serum miR-1915-3p and miR-455-3p as biomarkers for breast cancer. PLoS One. 2018;13(7):e0200716. https://doi.org/10.1371/journal.pone.0200716.

40. Xu CS, Li HH, Zhang L, Jia TJ, Duan L, Lu CR. MicroRNA-1915-3p prevents the apoptosis of lung cancer cells by downregulating DRG2 and PBX2. Mol Med Rep. 2016;13(1):505-12. https://doi.org/10.3892/mmr.2015.4565.

41. Li M, Makkinje A, Damuni Z. The myeloid leukemia-associated protein SET is a potent inhibitor of protein phosphatase 2A. J Biol Chem. 1996;271(19): 11059-62. https://doi.org/10.1074/jbc.271.19.11059.

42. Hung MH, Chen YL, Chu PY, Shih CT, Yu HC, Tai WT, et al. Upregulation of the oncoprotein SET determines poor clinical outcomes in hepatocellular carcinoma and shows therapeutic potential. Oncogene. 2016;35(37):4891902. https://doi.org/10.1038/onc.2016.21.

43. He K, Shi L, Jiang T, Li Q, Chen Y, Meng C. Association between SET expression and glioblastoma cell apoptosis and proliferation. Oncol let. 2016;12(4):2435-44. https://doi.org/10.3892/ol.2016.4951.

44. Liu H, Gu YX, Wang H, Yin J, Zheng G, Zhang Z, et al. Overexpression of PP2A inhibitor SET oncoprotein is associated with tumor progression and poor prognosis in human non-small cell lung cancer. Oncotarget. 2015; 6(17):14913-25. https://doi.org/10.18632/oncotarget.3818.

45. Hung MH, Wang C, Chen YL, et al. SET antagonist enhances the chemosensitivity of non-small cell lung cancer cells by reactivating protein phosphatase 2A. Oncotarget. 2016;7(1):638-55. https://doi.org/10.18632/ oncotarget.6313.

46. Alarcón RC, Lee H, Goodarzi H, Halberg N, Tavazoie SF. N6-methyladenosine (m6A) marks primary microRNAs for processing. Nature. 2015; 519(7544):482-5. https://doi.org/10.1038/nature14281.

47. Han J, Wang JZ, Yang X, Yu H, Zhou R, Lu HC, et al. METTL3 promote tumor proliferation of bladder cancer by accelerating pri-miR221/222 maturation in m6A-dependent manner. Mol Cancer. 2019;18(1):110. https://doi.org/10.11 86/s12943-019-1036-9.

48. Peng W, Li J, Chen R, Gu Q, Yang P, Qian W, et al. Upregulated METTL3 promotes metastasis of colorectal Cancer via miR-1246/SPRED2/MAPK signaling pathway. J Exp Clin Cancer Res. 2019;38(1):393. https://doi.org/1 0.1186/s13046-019-1408-4.

49. Wang H, Deng Q, Lv ZY, Ling Y, Hou X, Chen Z, et al. N6-methyladenosine induced miR-143-3p promotes the brain metastasis of lung cancer via regulation of VASH1. Mol Cancer. 2019;18(1):181. https://doi.org/10.1186/s12 943-019-1108-x.

50. Alarcón RC, Goodarzi H, Lee H, Liu XH, Tavazoie S, Tavazoie S. HNRNPA2B1 is a mediator of m6A-dependent nuclear RNA processing events. Cell. 2015; 162(6):1299-308. https://doi.org/10.1016/j.cell.2015.08.011.

\section{Publisher's Note}

Springer Nature remains neutral with regard to jurisdictional claims in published maps and institutional affiliations.

Ready to submit your research? Choose BMC and benefit from:

- fast, convenient online submission

- thorough peer review by experienced researchers in your field

- rapid publication on acceptance

- support for research data, including large and complex data types

- gold Open Access which fosters wider collaboration and increased citations

- maximum visibility for your research: over $100 \mathrm{M}$ website views per year

At BMC, research is always in progress.

Learn more biomedcentral.com/submissions 